Original Research Paper

\title{
Brain Computer Interface for Emergency Virtual Voice
}

\section{Arpitha $\mathbf{M J}^{1}$, Binduja ${ }^{1}$, Jahnavi $\mathbf{G}^{1}$, Kusuma Mohanchandra ${ }^{1}$}

${ }^{1}$ Department of Information Science and Engineering. Dayananda Sagar Academy of Technology and Management, Bangalore. Karnataka, India.

\section{Article History}

Received:

27.03.2021

Revised:

09.06.2021

\section{Accepted:}

16.06.2021

*Corresponding Author:

Arpitha MJ

Email

arpitha.jrm@gmail.com

This is an open access article, licensed under: $\mathrm{CC}-\mathrm{BY}-\mathrm{SA}$
Abstract: Alzheimer's disease (AD) is one of the type of dementia. This is one of the harmful disease which can lead to death and yet there is no treatment. There is no current technique which is $100 \%$ accurate for the treatment of this disease. In recent years, Neuroimaging combined with machine learning techniques have been used for detection of Alzheimer's disease. Based on our survey we came across many methods like Convolution Neural Network $(\mathrm{CNN})$ where in each brain area is been split into small three dimensional patches which acts as input samples for CNN. The other method used was Deep Neural Networks (DNN) where the brain MRI images are segmented to extract the brain chambers and then features are extracted from the segmented area. There are many such methods which can be used for detection of Alzheimer's Disease.

Keywords: BCI Applications, BCI Challenges, Brain Signal Acquisition, Feature Extraction, Mind Commands. 


\section{Introduction}

Brain Computer Interface (BCI) is the piece of hardware that allows humans to control or communicate with technology using the electrical signals generated in the brain. A speech BCI would make it possible to communicate in real time through neural correlations of attempted or imagined speech [1] that would potentially restore communication and improve the quality of life of enclosed patients and other patients with serious communication disorders

\subsection{History of BCI}

Formerly, research on BCI started at University of California in 1970. The mid 1990's lead to the discovery of first neuroprosthetic devices for humans which can augment nerves that are not working. The year 1998 marked a remarkable development in brain mapping field where the first brain computer interface object was implanted into a human being [2]. In December 2004, Jonathan Wolpaw and researchers at New York State Department of Health's Wadsworth Center proposed a research report that signifies the capability of BCI to control the computer. In this study, electroencephalography (EEG) signals from motor cortex (region of the cerebral cortex involved in planning and controlling movements) of subjects were captured by wearing a cap that contain electrodes. From that signals, features were extracted and converted into the outputs that replace, restore, enhance, supplement, or improve human functions. The various neural signals recorded noninvasively by $\mathrm{BCI}$ researchers during the trials that involve electroencephalography (EEG) [3], functional magnetic resonance imaging (fMRI), near-infrared spectroscopy (NIRS), magnetoencephalography (MEG), or invasively (e.g., electrocorticography).

\subsection{Types of BCI}

There are few types of BCI, namely:

1) Active $\mathrm{BCI}(\mathrm{BMI})$ : $\mathrm{BCI}$ that derives output from brain activity that's controlled directly by the user, independently of external events, to manage the applying [4].

2) Reactive BCI: BCI derives its outputs from brain activity resulting from external stimulation, which is indirectly modulated by users to manage the applying [5].

3) Passive or Affective BCI (BMI): It is a BCI that obtains its outcome from spontaneous cognitive function with no intention of voluntarism [6].

\subsection{Applications of $\mathrm{BCI}$}

The scope of BCI is vast. Initially most BCI's were developed for medicalapplications which ranges from detection and prevention to neurorehabilitation that compensates any functional alterations [7].

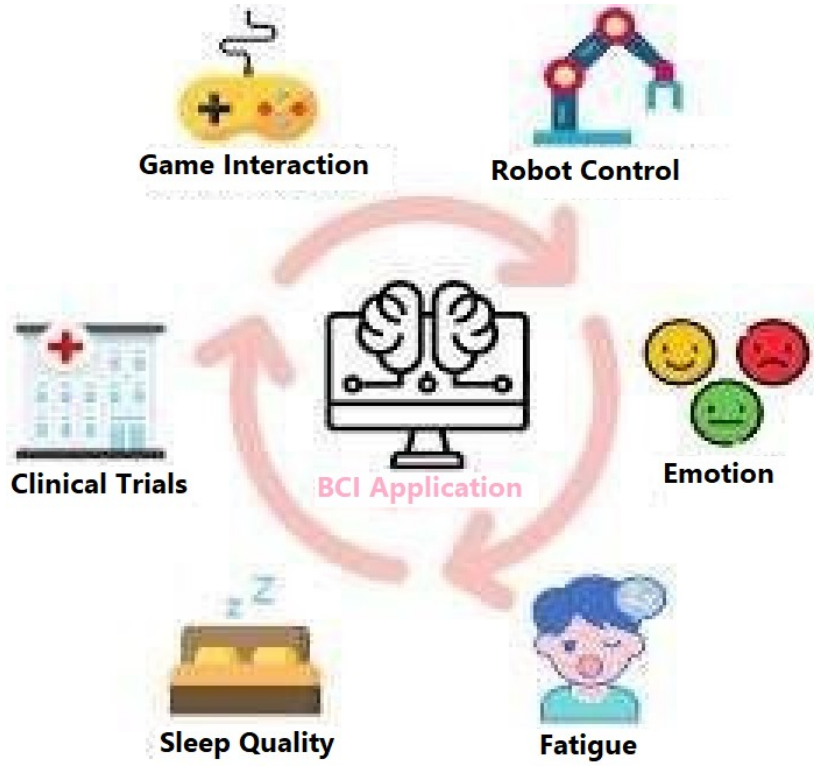

Figure 1. Applications of BCI 
Later on it started spreading its wings on various fields such as education, self-regulation, production, marketing, security as well as games and entertainment which focuses on neurorehabilitation after neurological diseases or injuries, Entertainment and gaming, lie detection, sleep-stage or mood monitoring, brain fingerprinting, trust assessment, monitoring pilots, air traffic controllers [8] and so on as shown in figure. In the last several decades, to provide an alternative communication tool for those with severe neuromuscular disorders (e.g., amyotrophic lateral sclerosis, spinal cord injury, and brainstem stroke) a great number of BCI systems have been developed. Researches on BCI is still continuing to open new doors.

\section{Brain Computer Interface}

Brain Computer Interface (BCI) is often used to resolve plenty of the impairments of disabled people [9]. BCI will provide access option for in dividuals with serious speech aswell as physic al impairments which helps to avoid the use of other interfaces. When used for communicati on, BCI systems can be described a creative an ugmentative andalternative communication (AAC) device. BCI can indeed be applied to a wide variety of tasks, not all of which are limited to neurofeedback, restoring motor function to paralyzed patients, allowing patients to communicate with locked patients and enhancing sensory processing. It works in three mainsteps: At first gathering brain signals, and afterwards analysing those signals and as per the obtained EEG [10] signals instructions are delivered to the connected machine.

For example, consider user answering YES or NO questions [11]. At first, he/she will read and analyse the question. Later, he/she memorise the answer (Yes or No depending on the question). The brain signals produced while memorising the answer are collected and analysed, later as per the obtained EEG signals YES or NO classification is done by applying appropriate algorithm. Then the Virtual voice of the output (YES/NO) comes out through speaker.

$\mathrm{BCI}$ provides direct data exchange between brain and computer, there are basically four important steps to be followed to control a BCI and later the brain signals are decoded accordingly as shown in Figure 1.

WORKING OF BRAIN COMPUTER INTERFACE(BCI)

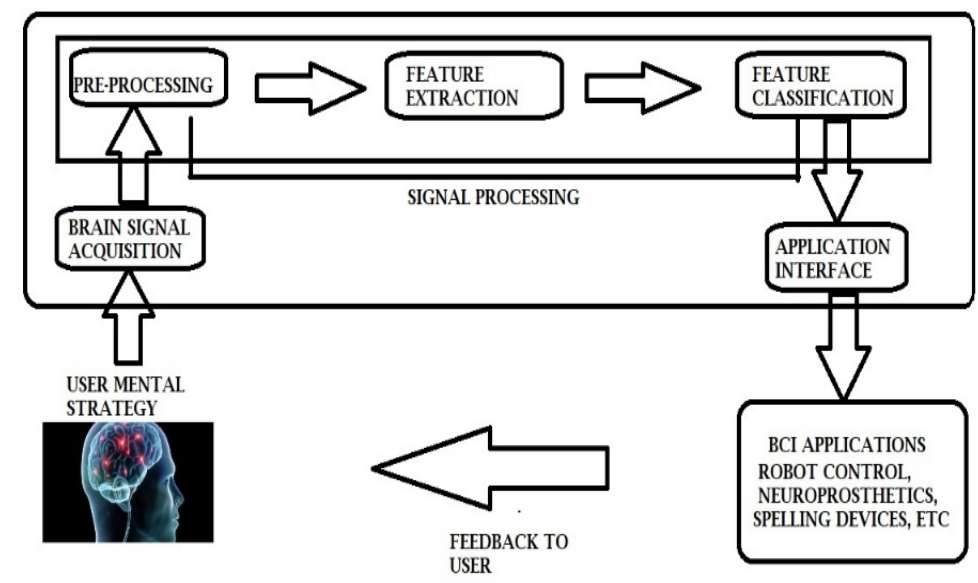

Figure 2. Steps Involved in BCI

At this step, through the electrodes we receive the raw brain signalsand start decoding process of the desired signals.

a) Pre-processing: From the signals, Internal and external noise must be eliminated and at this stage it is best to do so using a time filter. This move has a big effect on performance improvement.

b) Feature extraction and selection: In feature extraction, some features are extracted after eliminating noises from the brain signals. Using the required features, Performance can be 
dramatically improved. For feature extraction, several approaches are proposed which are CSP [12], discrete wavelet transform, power spectrum and AAR. In feature selection/ feature reduction, it is always better to use some methods because some features dimensions obtained from brain signals are very high which reduces efficiency [13].

c) Feature classification and post-processing: In this step, brain signal classification process takes place where the classifier will be trained with some features of brain signals and decide on a new motor imagery [14].

d) Virtual voice output: In the last step, the virtual voice of the words (memorised by the patient) will be produced from computer through the speakers [15].

e) Depiction is done through subjects imagining vowels like a, e, i, o, u. [16].

\section{BCI for Virtual Voice}

\subsection{Brief Study on BCI}

Humans controlling devices through thinking or brain signals may sound like something impossible but Brain Computer Interface is proving in the reality [17]. BCI is an emerging technology which is getting immense popularity in this current trend. This trend shows that an increasing number of researchers are becoming engaged in BCI science. The number of BCI articles is significantly getting increased as shown in Figure 3.

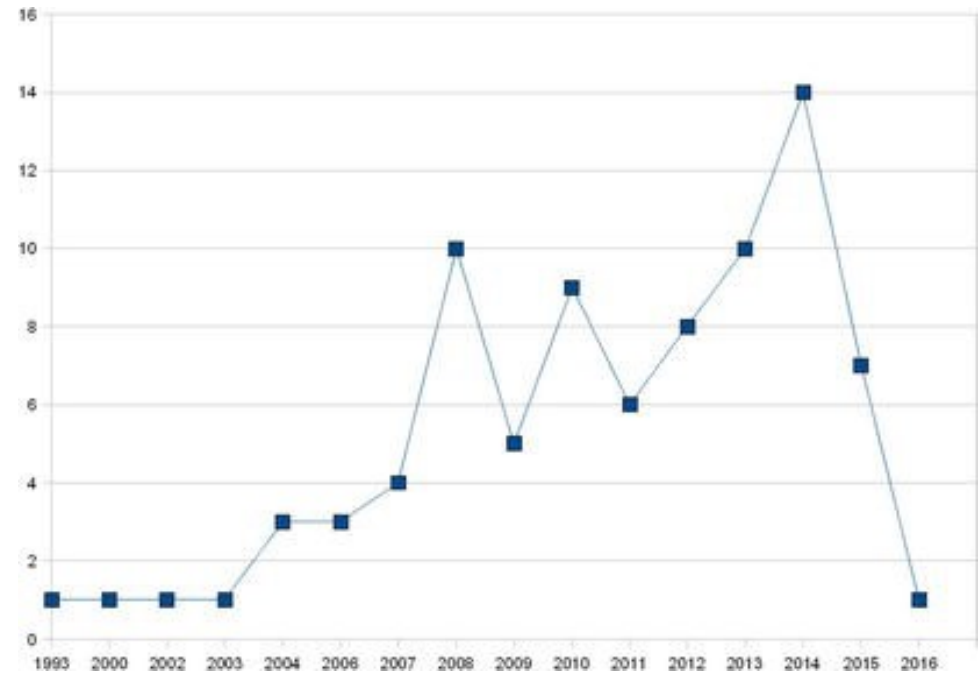

Figure 3. Increase in BCI articles

Figure 3 shows the increase in the BCI publications from 2000 to 2016. According to the picture below, initially BCI's were in not much demand and people thought controlling devices through brain was just impossible but gradually graph shows an increase in the publications of BCI wherein researchers started focusing to make impossible possible.

Figure 4 depicts pie charts showing the percentages of each brain-computer interface (BCI) paradigm used in EEG-based BCI articles published between 2007 and 2011.In this figure, "nonmotor mental imagery" means BCI paradigms that use non-motor imaging functions, e.g., mental navigation, sending SMS just by motor thinking, mental computation, internal singing, virtual voice through system, mental painting whereas " motor imagery" is a mental mechanism by which a person simulates a desired behaviour,"Visual P300" denotes is a general measurement of cognitive efficiency, "SSVEP" and "Auditory" denotes the usage of brain signals evoked by visual stimulus and auditory stimulus from brain by BCI paradigms. When two systems are integrated sequentially "Hybrid" is obtained. Percentage of motor imagery being used was higher in 2007(82\%) compared to 2011(44\%) but there was a significant increase in hybrid(0\%-6\%) and P300(4\% -24\%).On an average, usage of motor imagery was $56 \%$, visual P300 was $18 \%$,SSVEP $10 \%$, and rest sum up to $14 \%$.[3] 


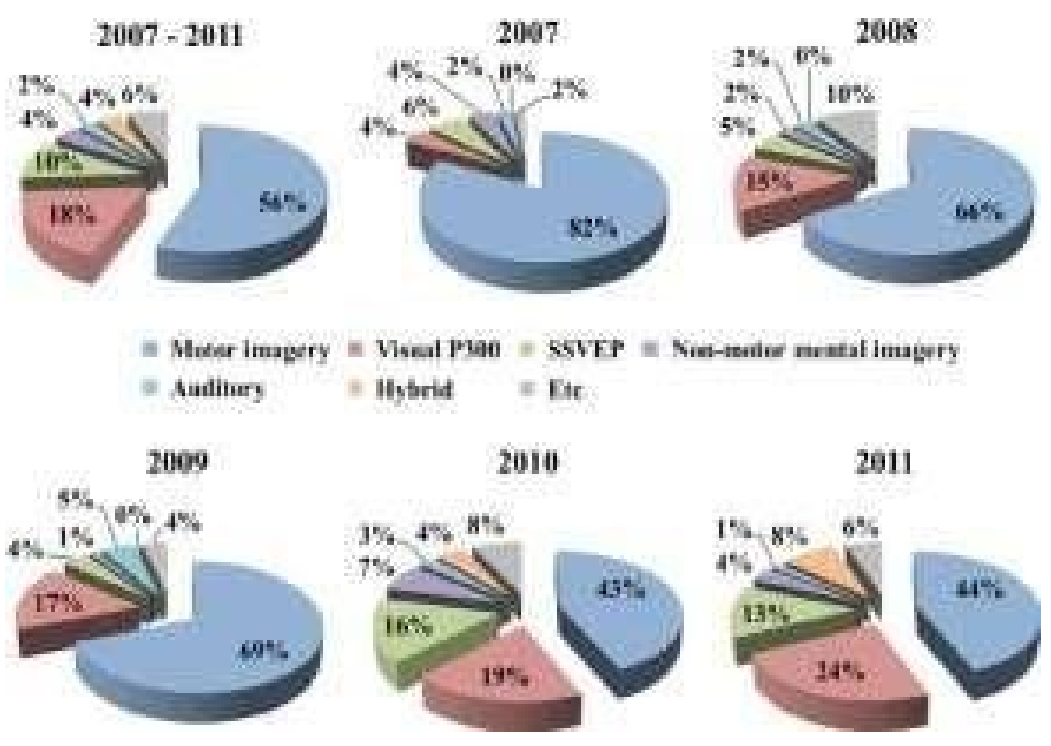

Figure 4. Percentage of BCI Paradigm used from 2007 and 2011

Today, technology is steadily shifting to the consumer market. Growing numbers of startups and big tech companies are focusing on reliabl e and cheaper BCIs.

\subsection{Bio-Signals for $\mathrm{BCI}$}

The average human brain comprises about 86 billion nerve cells called neurons. Neurons are the essential units of the nervous system Neurons interact by processing sensory feedback from the outer world and sending commands to our muscles using electrical signals. These signals are called the BioSignals which are used in BCI's for expected output. In order to perform the above operation, signals must be detected and this can be done in following ways. Bio signals are of two type, i.e. brain and non-brain signals.

\section{a) Brain Signals}

a. Electroencephalogram (EEG) and Magnetoencephalography (MEG)

An EEG is a test that monitors, identifies and documents brain wave patterns if there are any anomalies and MEG [6] provides high temporal andspatialresolutions.

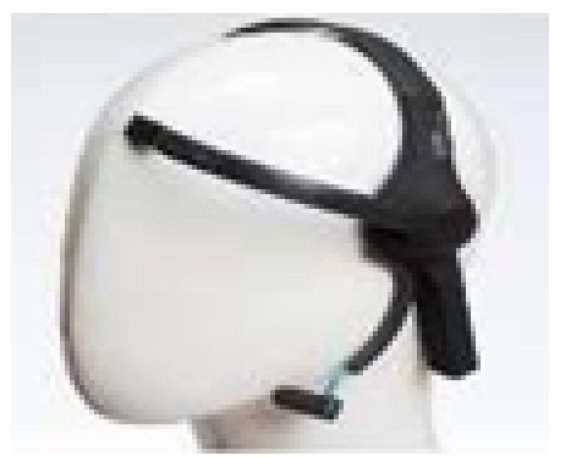

Figure 5. Neurosky Mindwave

b. The technique involves pressing small metal disks with electrodes on the scalp, and is used to track electrical impulses from the behavior of the brain cells, and the $\mathrm{n}$ appear in the form of a graph. 
Figure 5 depicts a Neurosky Mindwave through which signals are extracted and Figure 6 shows a graph which is produced when the electrical impulses [14] are tracked as mentioned.

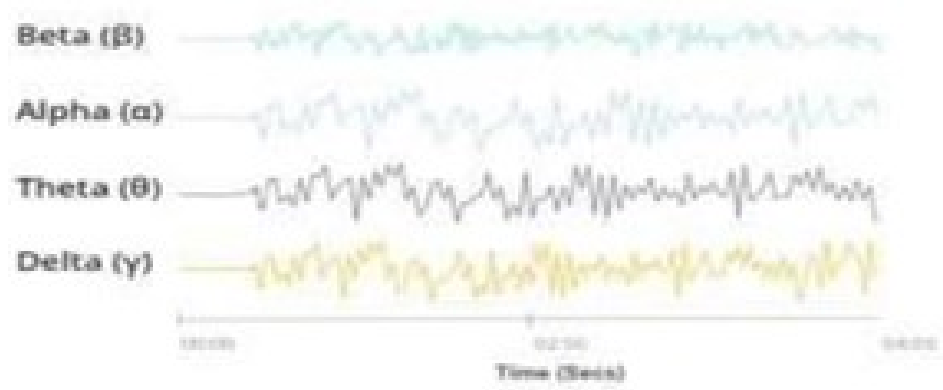

Figure 6. Electrical Impulses

c. Functional Near-Infrared Spectroscopy (fNIRS)

In context to BCI ,Functional near-infrared spectroscopy (fNIRS) is a wearable brainmonitoring technology used as functional neuroimaging where cortical hemodynamic responses are tracked, monitored and measured by near-infrared light placed over a scalp. FNIRS sensor pad which has light detectors and sources are used to acquire signals from right, central and left PFC [18].

d. Functional magnetic resonance imaging(fMRI)

Functional magnetic resonance imaging (fMRI) investigates the function of the brain, measures the symptoms, effects of paralysis by measuring differences in blood flow. fMRI is used to diagnose cognitive impairment that cannot be identified with other imaging methods.

b) Non-Brain Signals:

Electromyography (EMG), Electrocardiography (ECG), Electrooculography (EOG): Figure 7 depicts the signals being acquired from arm rather then brain which can be used for neural prosthesis [13].

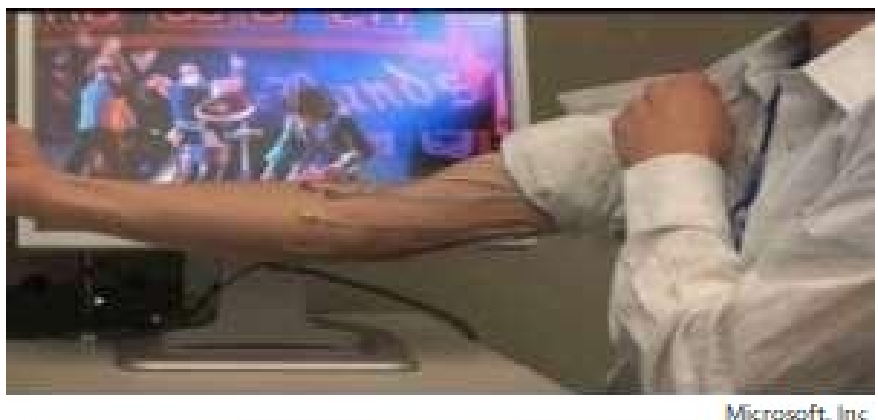

Figure 7. Arm Signals

\subsection{Target Application}

Anyone suffering from serious muscular disabilities such as ALS, stroke that causes paralysis, Locked-in Syndrome, tetraplegia, myasthenia gravis could be the goal applicant. For those individuals 
whose brain only operates and other body parts remain shut down, BCI for speech will provide communication ability (life saving words).

\subsection{Major BCI Components}

There are few major BCI components, namely:

1. Signal processing

Converting one signal to another. A BCI converts the input signal from the point of view of signal processing (e.g. EEG) into the control signal [19]. One can conveniently define BCI components as filters.Related classe $\mathrm{s}$ of filters are spatial filters, temporal filters, spatiotemporal filters, spectral filters, etc.

2. Feature Extraction

Off-the-shelf machine learning approaches frequently do not perform well when applied to raw signal fragments of the calibration recording! Too highdimensional (man why more parameters for fit)! Too complex structure to Catch (too much modelling freedom, requires domain-specific assumptions) Typical Solution: Implement additional mapping (called "feature extraction") from raw signal segments to feature vectors that extract the main features of raw observations. Output is generally of a lower dimensionality, let's hope that statistically "better" is distributed (easier to handle for machine learning).

3. Machine Learning

Most methodologies are consistent with a common framework of training and predictive function

\section{Conclusion}

From the literature study, we surveyed imagined speech based BCI papers and articles in order to investigate about the Brain Computer Interface that enables paralyzed With a high level impairment (the amount of function is substantial and the number of voluntary muscle control is markedly limited) to get a chance of conveying their basic needs as and when required and control devices through brain signals, learn methodologies including target applications, classification techniques and algorithms. It provides direct data exchange between brain and computer.

The development of BCI renders this technology feasible for patients suffering from neurological impairments causing to face many challenges in sensorimotor functions and communication with the environment. Therefore BCI's have increased demand for advanced, adaptive and personalized rehabilitation which is discussed above in the paper in terms of growth where we could find that BCI gets into mass market with relatively higher trend and also the publications on BCI is increasing dayby-day.

There are numerous applications of BCI in medical and non- medical fields out of which our work attempts to focus mainly on papers which detailed about providing virtual voice through the system which can be applied on the patients suffering from severe neuromuscular injuries or diseases.

We also could find that there are some series of steps to be followed to get the desired output and these steps include signal processing, feature extraction, classification, and output. All these steps are sequentially followed using algorithms accordingly. Based on the previous research, some studies extend to consideration of other BCI stakeholders such as medical science practitioners, caregivers or health care providers. The majority of current published worksare basically proof of concept studies. Thus, in the near future, BCI systems can become a new form of human-machine interaction with daily usage levels close to other current interfaces. Ultimately, non-muscular communication and control is no longer just imagination but the reality.

\section{References}

[1] C. Cooney, A. Korik, F. Raffaella, and D. Coyle, "Classification of Imagined Spoken WordPairs Using Convolutional Neural Networks. In the 8th Graz BCI Conference," Verlag der Technischen Universitat Graz, pp. 338-343, September 2019.

[2] E. K. Hachem and M. H. Harouni Alaou, "Improvement of the Intelligent Tutor by Identifying the Face of the E-Learner's", International Journal of Artificial Intelligence, vol. 6, no. 2, pp. 112 - 119, Dec. 2019. 
[3] X. Chi, J. B. Hagedorn, D. Schoonover, and M. D'Zmura, "EEG-Based Discrimination of Imagined Speech Phonemes," International Journal of Bioelectromagnetism, vol. 13, no. 4, pp. 201-206, 2011.

[4] S. Lee, M. Lee, J. Jeong and S. Lee, "Towards an EEG-based Intuitive BCI Communication System Using Imagined Speech and Visual Imagery," IEEE International Conference on Systems, Man and Cybernetics (SMC), Bari, Italy, 2019, pp. 4409-4414, doi:10.1109/SMC.2019.8914645. 2019

[5] B. Min, J. Kim, H. J. Park, and B. Lee, "Vowel Imagery Decoding toward Silent Speech BCI Using Extreme Learning Machine with Electroencephalogram," BioMed research international, 2016.

[6] D. Dash, A. Wisler, P. Ferrari, and J. Wang, "Towards a Speaker Independent Speech-BCI Using Speaker Adaptation," In INTERSPEECH, pp. 864-868, 2019.

[7] A. S. Abdulbaqi, I. Y. Panessai. "Efficient EEG Data Compression and Transmission Algorithm for Telemedicine," Journal of Theoretical and Applied Information Technology (JATIT), vol. 97, no. 4, 2019.

[8] A. S. Abdulbaqi, Y. S. Enas, A. D. M. Saif, and I. Y. Panessai. "Virtual Environments Utilization for ECG Signals Analysis and Evaluation: Towards Heart Condition Assessment," IOP Conference Series: Materials Science and Engineering, vol. 928, 2020.

[9] M. Kusuma, and S. Saha. "A Communication Paradigm Using Subvocalized Speech: Translating Brain Signals Into Speech," Augmented Human Research 1.1, 2016.

[10] K. Brigham, and B. V. Kumar, June 2010. "Imagined Speech Classification with EEG Signals For Silent Communication: A Preliminary Investigation into Synthetic Telepathy," IEEE.In 2010 4th International Conference on Bioinformatics and Biomedical Engineering, pp. 1-4, 2010.

[11] A. R. Sereshkeh, R. Trott, A. Bricout, and T. Chau. "EEG classification of covert speech using regularized neural networks", IEEE/ACM Transactions onAudio, Speech, and Language Processingvol. 25 no. 12, pp. 2292-2300, 2017.

[12] F. M. Zeki, and A. Hamadani. "Online Brain Computer Interface Based Five Classes EEG to Control Humanoid Robotic Hand," In 2019 42nd InternationalConference on Telecommunications and Signal Processing (TSP), IEEE, pp. 406-410, 2019.

[13] C. S. DaSalla, H. Kambara, M. Sato, and Y. Koike, Y. "Single-Trial Classification Of Vowel Speech Imagery Using Common Spatial Patterns," Neural networks, vol. 22, no. 9, pp. 13341339, 2009.

[14] A. S. Abdulbaqi, S. M. Najim, S. M. Al-barizinji, and I. Y. Panessai. "A Secured System for Tele Cardiovascular Disease Monitoring," Computational Vision and Bio-Inspired Computing. 209-222. 2021. Springer Singapore.

[15] A. K. Gopala, J. Chartier, and E. F. Chang. "Speech synthesis from neural decoding of spoken sentences," Nature vol. 568, no. 7753, pp. 493-498, 2019.

[16] S. SR, V. Desai, M. Singh, and K. M, "Survey on Early Detection of Alzhiemer's Disease Using Capsule Neural Network", International Journal of Artificial Intelligence, vol. 7, no. 1, pp. 7-12, Apr. 2020.

[17] P. Saha, M. Abdul-Mageed, and S. Fels. "Speak your mind! Towards imagined speech recognition with hierarchical deep learning," arXiv preprint arXiv, pp. 1904.05746, 2019.

[18] A. S. Abdulbaqi, S. M. Najim, S. D. Mahmood, and I. Y. Panessai. "A Tele Encephalopathy Diagnosis Based on EEG Signal Compression and Encryption," Springer Singapore, 2021.

[19] A. Manvi, A. Masood, and K. Mohanchandra, "Brain Operated Wheelchair Using a Single Electrode EEG Device and BCI", International Journal of Artificial Intelligence, vol. 7, no. 1, pp. 1-6, Apr. 2020. 